\title{
Analysis of mechanical properties of polylactic acid using a new 3D printer nozzle
}

\begin{abstract}
Additive manufacturing, also known as three-dimensional (3D) printing, is the process of developing 3D products in a layer-by-layer manner using filament as a material feedstock to create a solid structure. Owing to its unique properties and advantages, which include biodegradability and printing speed, polylactic acid is one of the most common 3D printing extrusion materials. While a considerable attention has been paid to the manipulation of process parameters in order to achieve desired finished product quality, to date less research has been performed on improving the hardware systems of low-cost 3D printers. This study focuses on fabricating the 3D printer nozzle parts, with an emphasis on die angle, nozzle diameter, liquefier design, and insulator composition. Modifying the properties of these components from the conventional nozzle, it is possible to optimize the stability and accuracy of the extrusion process, leading to better-quality printed products. To demonstrate the capability of the new nozzle, its tensile and compressive strengths were compared to those of a conventional nozzle. The obtained results proved that the proposed augmentations to the nozzle system lead to finished products with improved mechanical properties.
\end{abstract}

Keyword: 3D printer nozzle; Fused deposition modeling; Open-source 3D printer; Polylactic acid 